\title{
Spatio-Temporal Building Population Estimation for Highly Urbanized Areas Using GIS
}

\author{
Konstantin Greger \\ Division of Spatial Information Science, University of Tsukuba
}

\begin{abstract}
Detailed population information is crucial for the micro-scale modeling and analysis of human behavior in urban areas. Since it is not available on the basis of individual persons, it has become necessary to derive data from aggregated census data. A variety of approaches have been published in the past, yet they are not entirely suitable for use in the micro-scale context of highly urbanized areas, due mainly to their broad spatial scale and missing temporal scale. Here we introduce an enhanced approach for the spatio-temporal estimation of building populations in highly urbanized areas. It builds upon other estimation methodologies, but extends them by introducing multiple usage categories and the temporal dimension. This allows for a more realistic representation of human activities in highly urbanized areas and the fact that populations change over time as a result of these activities. The model makes use of a variety of micro-scale data sets to operationalize the activities and their spatio-temporal representations. The outcome of the model provides estimated population figures for all buildings at each time step and thereby reveals spatio-temporal behavior patterns. It can be used in a variety of applications concerning the implications of human behavior in urban areas.
\end{abstract}

\section{Introduction}

Detailed population information is crucial for the micro-scale modeling and analysis of human behavior and processes in urban areas. Ideally it should be based on individual persons, yet for privacy reasons such data are generally not available. Therefore it has become necessary to derive estimated data from aggregated data sets such as census data.

Wu et al. (2005) provided an extensive summary of a variety of approaches and methodologies published in the past. Since this has now become somewhat outdated, we first provide a brief overview of some of the major achievements in the field of population estimation methodologies and focus on those studies that have been the most influential in the development of the approach we introduce in this article.

Tobler et al. (1997) suggest their Gridded Population of the World as a macro-geographic approach for population estimation on a global scale. They argue that "the average daily activity space of individuals is dependent upon culture, environment, social, and urban-rural status, but averages more than $15 \mathrm{~km}$ in western societies" (Tobler et al. 1997, p. 207). In

\footnotetext{
Address for correspondence: Konstantin Greger, Division of Spatial Information Science, University of Tsukuba, 1-1-1 Tennodai, Tsukuba, Ibaraki 305-8572, Japan. E-mail: greger@geoenv.tsukuba.ac.jp

Acknowledgements: This research was partially funded by the 2012 Sinfonica Statistical \& GIS Research Grant of the Statistical Institute for Consulting and Analysis. Also, we thank the Center for Spatial Information Science at the University of Tokyo for providing several data sets within the scope of joint research No. 405: People Flow data from 2008 for the Tokyo Metropolitan Area provided by the CSIS People Flow Project Office; Zmap-TOWN II 2008/09 Shape files for the Tokyo Metropolitan Area as well as Telepoint Pack DB February 2011 provided by Zenrin Co., Ltd.; 2010 population census data as well as 2009 business census data and the national census map data provided by the Statistical Institute for Consulting and Analysis (Sinfonica). Lastly, this manuscript has benefited from comments from a number of internal and two anonymous external reviewers whose valuable insights and comments we greatly acknowledge.
} 
their calculations they rely on population data aggregated on secondary administrative levels and ultimately produce worldwide population figures "interpolated to a 5 - by 5 -minute grid using a smoothing algorithm developed by Tobler (1979)" (Sutton et al. 2003, p. 546).

The LandScan Global Population Project at the Oak Ridge National Laboratories (Dobson et al. 2000; Bhaduri et al. 2007) also attempts to provide worldwide population figures, albeit on a finer spatial scale of $30 \times 30$-second grid cells and more recently even with a $90 \times 90 \mathrm{~m}$ resolution for the USA. They do this by improving the pycnophylactic and dasymetric interpolation algorithms that had traditionally been employed, using additional ancillary spatial data such as roads, slope, land use, urban areas, nighttime lights (Elvidge et al. 1997; Sutton et al. 2001), and coastlines. In addition, they incorporate diurnal nighttime and daytime populations to account for the variations in population distributions as a result of different underlying activities.

Similarly, McPherson and Brown (2004) focus on the shifts in populations in their work at the Los Alamos National Laboratory. They argue that "the 1-kilometer resolution used in that [i.e. the LandScan] dataset is insufficient for urban exposure analyses" (McPherson and Brown 2004, p. 2) and provide nighttime residential, daytime residential, and daytime workplace population data on a $250 \times 250 \mathrm{~m}$ grid for the continental US and Hawaii. In their model they rely on a number of data sets, including nighttime population census data, a business directory, and a "Census County to County Journey to Work" data set by the US Census Bureau containing the numbers of people moving from their homes to their workplaces. This approach bears some similarity to the one we introduce in this article, although it is more simplistic and on a coarser spatial scale.

Along the same lines, Martin (1996) and Martin et al. (2009) developed a model for the estimation of "24-hour gridded population models of the UK [. . .] based on an existing adaptive kernel density approach for building gridded population models (Martin 1996), which is now being extended to become a spatio-temporal kernel density estimation method" (Martin et al. 2009, p. 1). To our best knowledge, this marks the first attempt to overcome the diurnal model of daytime and nighttime populations and provide an insight into the differences in population distributions over the course of a day. They achieve this by not relying exclusively on the available census data, but by incorporating additional secondary data sets, such as employee numbers, traffic and passenger flow data, as well as counts of prison inmates, hospitalized people and tourists (Martin et al. 2009, p. 3). This is a fundamental parallel to the methodology we present in this article, but our approach overcomes the limitations regarding the availability of dynamic population data that Martin and colleagues deplore.

Ahola et al. (2007) attempt to overcome the coarse spatial resolutions of the aforementioned studies, which preclude their application in the micro-scale context of highly urbanized areas. They were able to do this by using population census data on the building level for their case study. In order to represent the dynamic characteristics of the population distribution they employed a spatial decision support framework (Malczewski 1999) using several data and a priori assumptions: "(1) basic static data on the population and infrastructure (municipal and governmental registers); (2) time-series data on the trends in various phenomena (data from the statistics); (3) spatio-temporal knowledge (spatio-temporal model of population); and (4) strategies on preparedness (governmental statements about the threats and the preparedness for them)" (Ahola et al. 2007, p. 938). They identified 14 individual time periods over the course of a week as a result of the modeled activities, but mention that "more detailed information about the temporal behaviour of different population groups could also improve the quality of the model" (Ahola et al. 2007, p. 952). This is one of the major aspects we pursued with the development of the model introduced in this article. 
As Martin et al. (2009, p. 1) remark, "grid-based population models have considerable advantages for population representation, offering more meaningful representation of settlement and neighbourhood pattern, including the geography of unpopulated areas, and providing stability through time. As a result, gridded models have seen extensive use where population must be integrated with environmental phenomena". On the downside, these grids are normally too generalized in their spatial resolution to be able to adequately represent facts and processes within highly urbanized areas. Another shortcoming of the models introduced above is the temporal scale, which is either left out completely or defined as a two-stage process that opposes the situations during the day and at night. A notable exception is the approach by Martin and colleagues (2009), but their assumption of all people of a certain demographic group being engaged in a certain activity (and hence being present at certain corresponding locations) at a certain time appears to us to be too generalizing, which again makes spatio-temporal micro-scale analyses unreliable.

Two approaches have been published recently that attempt to ameliorate these two shortcomings. Lwin and Murayama (2009) suggested a variety of calculation methods to estimate populations on a building basis, including areametric and volumetric approaches. In addition, Horanont (2012) suggested and exemplified the use of person trip data in the modeling of dynamic populations.

In this article we present a novel approach for a spatio-temporal micro-scale population estimation on a building basis. We incorporate multiple data sets, namely population census data, employment data, address point data and, following Horanont's (2012) suggestion, movement data. As a result, the model provides fine-grained results of the estimated populations within different usage categories for each building on a given timescale.

In Section 2 of this article we explain and discuss the basic methodology, which builds the foundation for the enhanced approach. Section 3 describes the extensions we made to the model and the data needed to perform the estimation process. We then go on to explain in detail the newly introduced dimensions of usage categories (Section 3.1) and temporal fluctuations derived from movement data (Section 3.2) in the course of a case study. In Section 4 we discuss the validation of the model, as well as its output and possible use cases, before summarizing and pointing out some shortcomings in the final section.

\section{Underlying Estimation Approach}

The population estimation approach we introduce in this article is an extension of the article by Lwin and Murayama (2009). There the authors introduced both areametric and volumetric methods for the estimation of residential building populations.

Both methods assume an equal distribution of the population on the available total floor space within all the residential buildings in a study area, but they differ in the method of deriving this total floor space area: while the areametric method refers exclusively to the buildings' footprint areas, the volumetric method also takes into account the number of floors per building. The former is due to the possible unavailability of comprehensive data about the number of floors per building. While it still allows for an estimation of the building populations, the authors remark that " $[t]$ he Areametric method is suitable for low-rise buildings especially in rural areas while the Volumetric method is suitable for high-rise buildings, especially in downtown areas" (Lwin and Murayama 2009, p. 404), thereby formulating one of the shortcomings of their proposed estimation approach. Another one would be the exclusive focus on residential buildings, which neglects all other building uses, such as offices, shops, schools, etc. 
The underlying assumption was an equal distribution of the population over the available total floor space, which allows for a sufficiently precise estimation of building populations. The limitations in their suggested method regarding the usage types (the authors only accounted for the residential population), and the missing temporal dimension inspired us to extend and further develop their basic approach into an enhanced methodology that can represent more realistically the underlying real-world processes that are the result of human actions, especially in urban areas.

\section{Enhanced Estimation Methodology}

The volumetric building population estimation methodology introduced by Lwin and Murayama (2009) uses Equation (1) to calculate population figures for all buildings. They point out that buildings with a nonresidential use and reasonably small footprint areas have to be excluded from the calculation. They also statistically derive the optimal minimum footprint area.

$$
B P_{i}=\left(\frac{C P}{\sum_{k=1}^{n} B A_{k} \cdot B F_{k}}\right) \cdot B A_{i} \cdot B F_{i}
$$

where $B P_{i}$ is the estimated population of building $i, C P$ is the population of the aerial unit that contains building $i, B A_{i}$ is the footprint area of building $i, B F_{i}$ is the number of floors of building $i$, and $n$ is the number of buildings that meet the user-defined criteria regarding residential usage and minimum footprint area and that are located inside the same aerial unit as building $i$ (modified from Lwin and Murayama 2009, p. 403).

From this formula it becomes obvious that, as well as the building information regarding footprint area and the number of floors, population data are also needed. These data can be obtained from a regular population census data set, available in most parts of the world. The aerial unit refers to the level of aggregation of the underlying population data. It depends on the data set used and can range from large-scale units like cities or quarters to smaller units like census tracts or even micro-scale units like census blocks or building blocks. For the sake of simplicity we will refer to these spatial entities as "census tracts" throughout this article, since this is the level of the data we used in our case study (cf. Section 3).

While their general availability is a positive aspect of population census data, two obvious shortcomings are its exclusive focus on residential populations, discounting other activities such as working or studying, and its missing temporal scale. Many authors have emphasized how census data do not portray the actual population distribution, but can, at their best, only represent the so-called "nighttime population" (Schmitt 1956; Dobson et al. 2000; McPherson and Brown 2004; Ahola et al. 2007; Bhaduri et al. 2007; Martin et al. 2009). In this context, Wu et al. (2005, p. 70) note that census data are "mainly concerned with residential populations and the daytime population distribution can be very different from that described by the census". Thus the data will be especially misleading in areas with minor residential use and a great number of other usages. Prime examples are highly urbanized city centers, which have undoubtedly high populations during daytime, but almost no residents. Whether or not they are completely deserted during the night depends on the presence of employees at night, for example due to globalized business activities at all times, and the existence of other 




Figure 12010 census data population density for the eastern Tokyo Metropolitan Area including the southern part of Saitama prefecture and the northern part of Kanagawa prefecture

land uses, such as entertainment facilities. Figure 1 shows a map of the central part of the Tokyo Metropolitan Area with its residential population density figures according to the 2010 census data. It is obvious how the population density in the core city (where our study area is located) is significantly lower than in the surrounding suburban belts, even reaching into the neighboring prefectures of Saitama to the north and Kanagawa to the south.

It is imperative to amend these two shortcomings in order for micro-scale population data to be "useful in hazard planning and response, environmental impact assessment, transportation planning, economic decision-making, and numerous other applications" as Sutton et al. (2003, p. 545) note. They go on to argue that "[u]seful measures of population density must be made at appropriate, application-specific, spatial and temporal scales" (Sutton et al. 2003, p. 545). Hence it was necessary to utilize additional data sets to incorporate both the multiple usage categories and the inherent temporal differences that affect building populations in highly urbanized areas in our enhanced model.

Firstly it was imperative to define all activities that are pursued in urban areas. Table 1 explains the activities we used in this article. We emulated the classifications by Axhausen 
Table 1 The six usage categories used in the model

\begin{tabular}{|c|c|c|}
\hline & Category & Address point usages (2,208 in total) \\
\hline 1 & home & Private households \\
\hline 2 & $\begin{array}{l}\text { business \& } \\
\text { office }\end{array}$ & $\begin{array}{l}\text { All types of offices and places of manual labor (e.g. factories, } \\
\text { agricultural, forestry, and fishery) excluding those aimed } \\
\text { predominantly at service tasks }\end{array}$ \\
\hline 3 & education & $\begin{array}{l}\text { Kindergarten, elementary schools, junior high schools, senior high } \\
\text { schools, vocational schools, schools for the disabled, universities, } \\
\text { research institutes, libraries }\end{array}$ \\
\hline 4 & retail \& service & $\begin{array}{l}\text { All types of shops, department stores; all types of service institutions } \\
\text { (e.g. gas stations, cleaning shops, branch banks, post offices, etc.) }\end{array}$ \\
\hline 5 & leisure \& hotel & $\begin{array}{l}\text { Restaurants, coffee shops, entertainment facilities (e.g. bars, movie } \\
\text { theaters, concert halls, etc.), sports facilities, hotels }\end{array}$ \\
\hline 6 & public institution & $\begin{array}{l}\text { Police departments, fire departments, hospitals, clinics, nurseries, } \\
\text { public assembly halls }\end{array}$ \\
\hline
\end{tabular}

et al. (2002), Bowman and Ben-Akiva (2001), and Jiang et al. (2012) in the definition of these six activities. Since these activities have to be mapped to multiple data sets from different sources later on in the modeling process, we formulated them as generally as possible while keeping intact their distinct characteristics. To derive them we used a data set of address points, which contained not only the spatial location but also an indicator of the usage for each data point. Starting from a total of 2,208 indicators we aggregated them to these six categories.

All data sets necessary for the estimation calculation we propose here together with their required attributes are shown in Table 2. It also shows the actual data sets we used in our building population estimation example in Tokyo, Japan. The study area comprises an area of roughly $43 \mathrm{~km}^{2}$ near Tokyo Station, namely the three wards of Chiyoda (including the Imperial Palace in its center), Chuo and Minato (including a part of the man-made island of Odaiba in Tokyo Bay) in central Tokyo (Figure 1). We selected this study area as it is one of the most highly urbanized areas in the world while at the same time very recognizable for its many different building types and diverse land uses. These building types and land uses also have a very strong effect on the distribution of the population over time: since the activities pursued here vary greatly between different times of the day, so do the associated population densities and distributions.

Equation (2) shows the enhanced spatio-temporal building population estimation methodology we are proposing here in a formalized fashion:

$$
B P_{i, c, t}=\left(\frac{A P_{A_{i, c, t}}}{\sum_{k \in A_{i, c}} B A_{k} \cdot B F_{k}}\right) \cdot B A_{i, c} \cdot B F_{i}
$$

where $B P_{i, c, t}$ is the building population of building $i$ in category $c$ at time $t, A P_{A i, c, t}$ is the total population of category $c$ at time $t$ of the census tract that contains building $i, A$ is the set of all census tracts, $B A_{i}$ is the footprint area of building $i, B A_{i, c}$ is the footprint area of building $i$ in category $c$, and $B F_{i}$ is the number of floors of building $i$. 
Table 2 Necessary data sets for the spatio-temporal building population estimation methodology and data sets used in this case study

\begin{tabular}{|c|c|c|c|}
\hline Data set & Attributes & Data set name and source & Date \\
\hline $\begin{array}{l}\text { Building } \\
\text { data }\end{array}$ & $\begin{array}{l}\text { Footprint area } \\
\text { Number of floors }\end{array}$ & $\begin{array}{l}\text { Zmap-TOWNII by Zenrin } \\
\text { Co., Ltd. }\end{array}$ & $2008 / 09$ \\
\hline \multirow[t]{2}{*}{$\begin{array}{l}\text { Census } \\
\text { data }\end{array}$} & $\begin{array}{l}\text { Residential populations (i.e. } \\
\text { common population census } \\
\text { information) }\end{array}$ & $\begin{array}{l}\text { Population census by the } \\
\text { Statistics Bureau at the } \\
\text { Ministry of Internal Affairs } \\
\text { and Communications }\end{array}$ & 2010 \\
\hline & $\begin{array}{l}\text { Employment populations (i.e. } \\
\text { business or economic census } \\
\text { information) }\end{array}$ & $\begin{array}{l}\text { Employment census by the } \\
\text { Statistical Institute for } \\
\text { Consulting and Analysis }\end{array}$ & 2009 \\
\hline $\begin{array}{l}\text { Address } \\
\text { point } \\
\text { data }\end{array}$ & $\begin{array}{l}\text { Spatial location of each address } \\
\text { point } \\
\text { Category of the person or } \\
\text { business represented by each } \\
\text { data point as shown in Table } 1\end{array}$ & $\begin{array}{l}\text { Telepoint Pack! by Zenrin } \\
\text { Co., Ltd. }\end{array}$ & 2011 \\
\hline $\begin{array}{l}\text { Population } \\
\text { movement } \\
\text { data }\end{array}$ & $\begin{array}{l}\text { Spatial location of each individual } \\
\text { at each time step } \\
\text { Trip purpose/activity } \\
\text { Means of transportation }\end{array}$ & $\begin{array}{l}\text { PersonFlow data by the } \\
\text { University of Tokyo Center } \\
\text { for Spatial Information } \\
\text { Science }\end{array}$ & 2008 \\
\hline
\end{tabular}

The calculation process itself is split into two main parts: first the categorical volumetric building population estimation process, which estimates the building population per usage category. This part of the estimation process, which we show in Section 3.1, produces meaningful results on its own, if the additional temporal dimension is not of interest or the necessary data are not available. In this case, Equation (2) has to be applied without the notation of the time $t$, producing $B P_{i, c}$ as the population of building $i$ in category $c$. The actual spatio-temporal volumetric building population estimation process, which includes the temporal dimension, is explained in detail in Section 3.2.

\subsection{Categorical Volumetric Building Population Estimation Process}

We first assigned the total number of address points as well as the number of address points in each of the six usage categories to each building. Figure 2 shows that the different usage categories are not distributed equally within the study area and instead reveal several patterns of residential clusters, an office cluster, as well as retail and leisure clusters. Due to the mix of usage categories prevalent not only over space but even within buildings in highly urbanized areas, it was necessary to implement these mixed uses in the estimation model. An analysis of the data set used in the case study revealed that $35 \%$ of all buildings contained address points of more than one category.

Since detailed information about the actual floor space occupancy per category within a building is usually not available, the model uses the relative ratio of address points per category per building as an approximation. Figure 3 shows examples of this relative ratio of usage for the categories: (1) "home," (2) "business \& office," and (3) "retail \& service” for a small portion of the study area. 


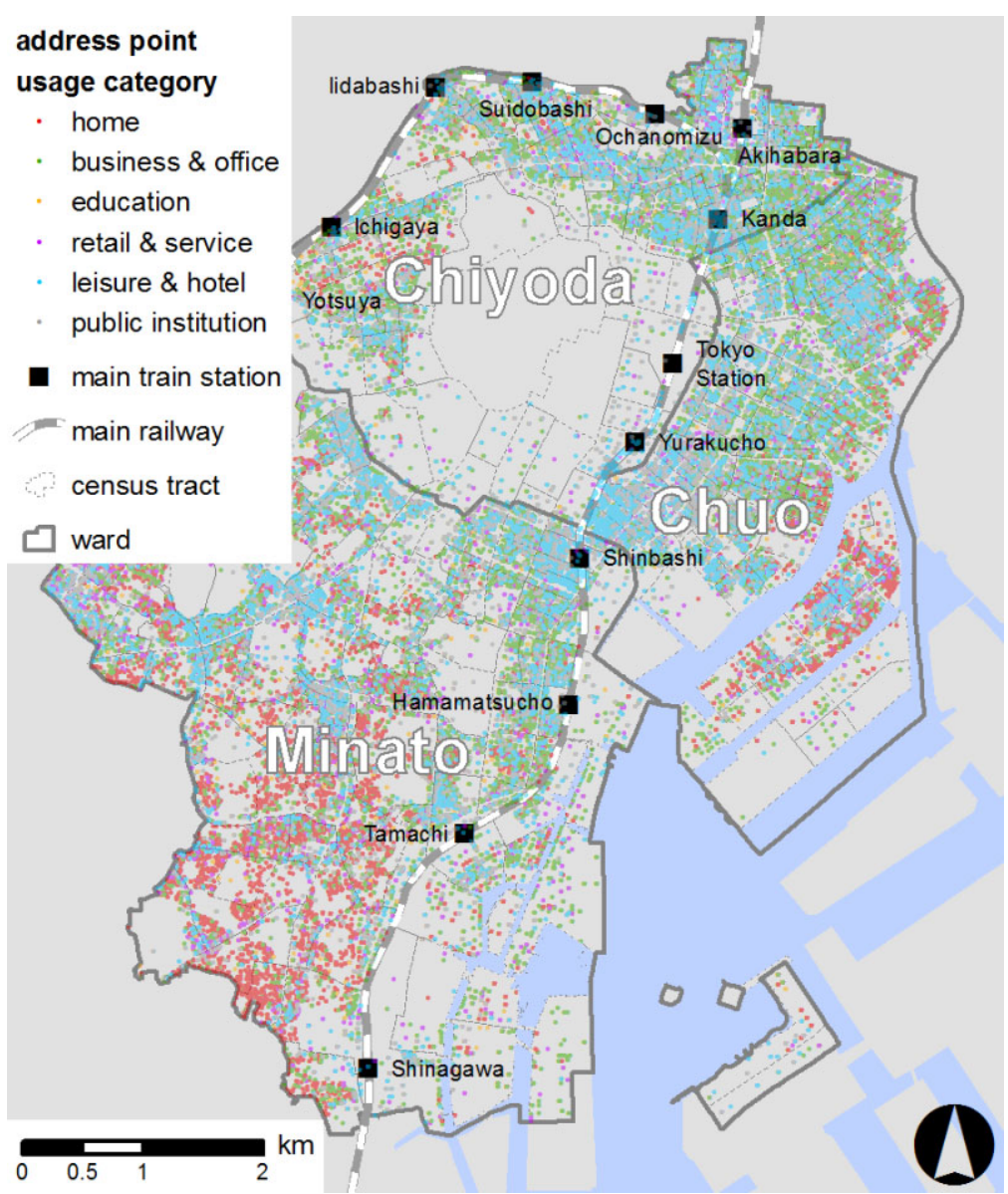

Figure 2 Spatial distribution of address points color-coded by their usage categories

Next we derived the total floor space in $\mathrm{m}^{2}$ for each building from the footprint area and the number of floors, as suggested in the preliminary approach. In addition, we were able to calculate the floor space occupied by each usage category within each building, using both the information about the total floor space and the relative ratio of usage per category within each building. This introduces two possible errors that will ultimately also affect the building population figures. Firstly, missing address point data can skew the floor space percentages per usage categories significantly. Secondly, our approach assumes an even split of the available floor space area within a building between the existing usage categories. This can be especially problematic in cases where one category occupies a larger portion of the space than others. While we are aware of these shortcomings we were unable to ameliorate them given the available data.

In order to match the floor space per category per building with the categorical population data as introduced in the original estimation model we then calculated the total floor space per category for all buildings within each census tract. Thereby we also relied on the aforementioned assumption of assigning the population of each census tract to the buildings it contains according to their ratio of floor space in the cumulative floor space of all buildings within it. Our enhanced model does this separately for each of the six usage categories. 

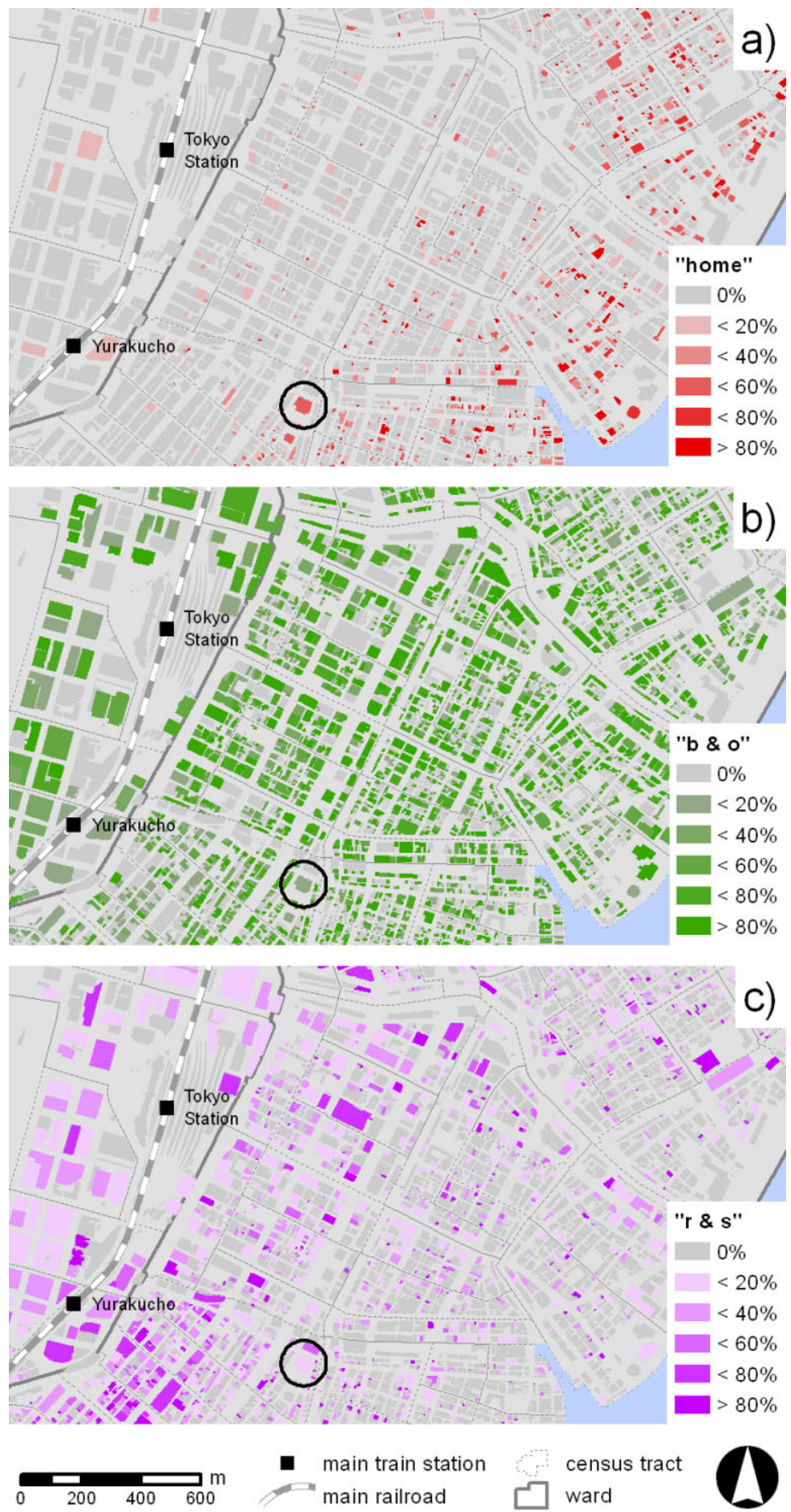

Figure 3 Maps of a detail of the study area, showing the relative floor space ratio per usage category for the categories: (a) "home"; (b) "business \& office"; and (c) "retail \& service" 
To achieve this we used different data sources for the calculation of the populations within each usage category: while regular population census data represents the residential population, also known as the "nighttime population," the employment census contains information about the number of employees in different business categories. We generalized the 16 employment categories defined by the Ministry of Health, Labour and Welfare in the original data set and grouped them to the five nonresidential usage categories we derived above from the 2,208 usages in the address point data set.

This allows us to put the number of employees per category per census tract into context with the relative floor space ratio of each category across each building within this census tract. Accordingly, the aforementioned building population estimation formula has to be employed once for the residential population (usage category "home") and once for each of the five remaining categories ("business \& office," "education," "retail \& service," "leisure \& hotel," "public institution") using the respective population figures and floor space ratios. As a result, we can then assign an estimated population per usage category to each building. In this context it should be mentioned that the current estimation model only accounts for the number of employees within the buildings, not for the number of people pursuing other activities there. Since the number of employees per category varies both per building and per census tract, it is necessary to perform the aforementioned calculations for all five categories (plus once for the residential population). This way the connection between the address point data set and the residential and employment census data sets is maintained throughout the model. In this context it should also be mentioned that due to the separate calculation processes for the different usage categories, the spatial representations ("census tracts") of the underlying population data sets do not necessarily need to be identical and can vary in the level of generalization. This is true, since all upcoming steps of the modeling process will work on the building level. Other, more transient populations like customers, guests, and visitors cannot be estimated in this fashion, and are therefore left out of the calculation. The student populations are a special case, since these can theoretically be estimated using the same methodology if data such as an "education census," which contains the number of students per census tract, should be available. For the case study in this article, however, we did not include these data.

We would also like to point out here another shortcoming of our estimation process, a result of the available data sets. With the assignment of residents and employees to the buildings based on the percentage floor space ratio within their respective census tract, we assume that all occurrences of a usage category accommodate an equal number of residents and employees per $\mathrm{m}^{2}$. As in reality there are quite big variations in the space requirements, even within usage categories, this can potentially introduce significant errors. With the available data we have not been able to circumvent this shortcoming.

In a last step these six categorical building population figures can then be added up to the total estimated building population of each building. Figure 4 shows the result of this final step.

This number can be understood as the estimated total building population, representing the maximum number of people that, according to the underlying assumptions and data sources used in the modeling, are present within each respective building. Yet this number probably does not reflect reality, as can be easily understood by the example of a building containing both residential and commercial use: while the residents are likely to leave their homes during the course of a day and tend to return in the evening, the employees would rather enter the building in the morning and leave in the late afternoon (as we will show in the upcoming section). As a result it is highly unlikely that $100 \%$ of the populations of both categories would be present in the building at the same time, rendering the current model output 


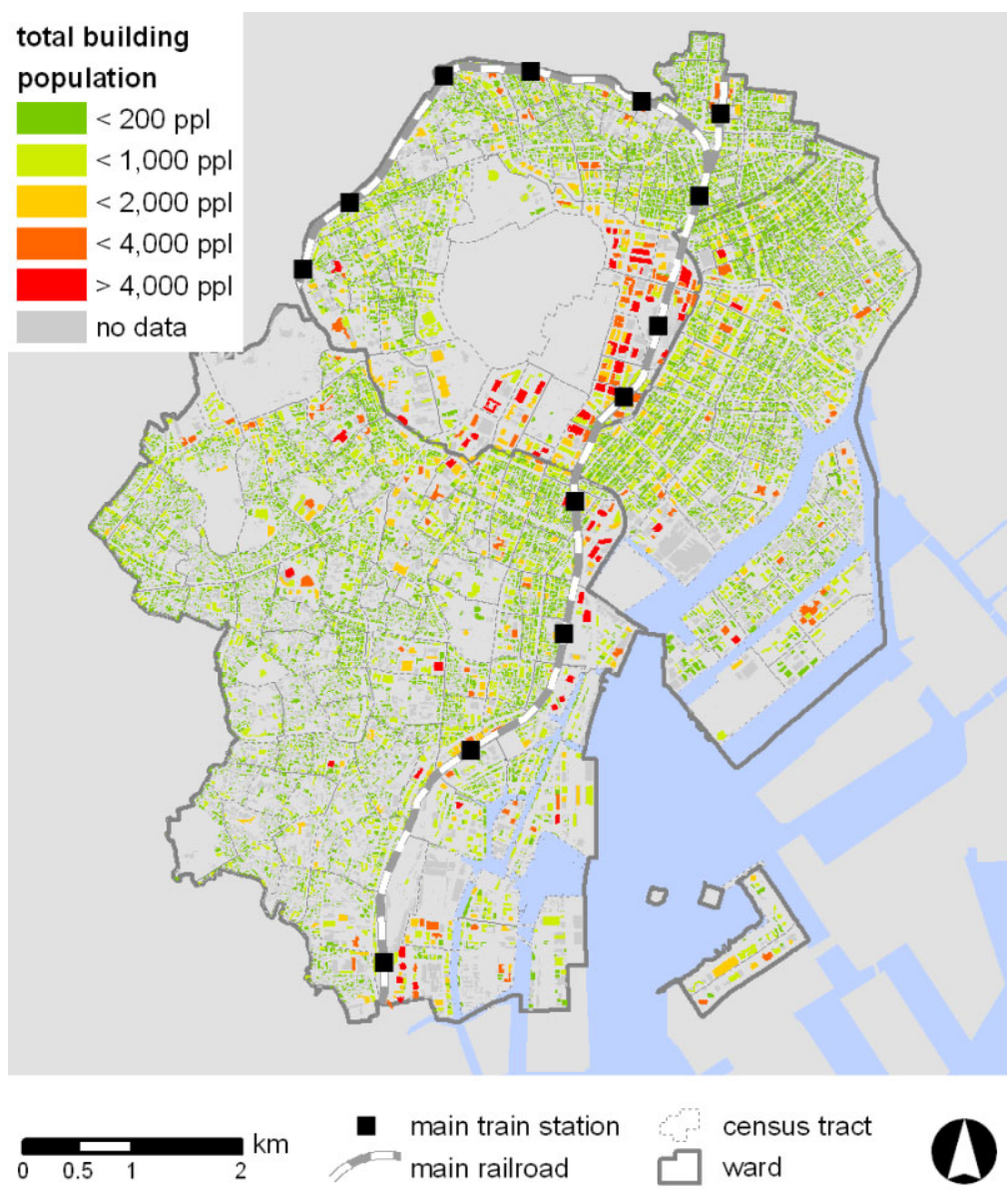

Figure 4 Map of the total estimated building population for each building in the study area

questionable, since it grossly overestimates the actual building population. It is therefore imperative to include a temporal dimension in the estimation process. In the next section we will describe this temporally enhanced process in detail.

\subsection{Adding the Temporal Dimension}

The outcome so far extends the original methodology by Lwin and Murayama (2009) by the introduction of five additional usage categories over their one-dimensional approach to residential populations. As mentioned above, this does not account for the fact that populations are not stationary over time, but move in space according to the routine activities performed by people in the urban space.

To account for this it is necessary to obtain spatio-temporal data representing the movements of people in the respective study area. In the case of this article we included one additional data set, which contains movement data of more than 600,000 individuals in the Greater Tokyo Metropolitan Area. The underlying data were originally collected using paper questionnaires and contain not only the locations and time stamps of the start and end of 
trips, but also the means of transportation, the purposes of the trips, and several sociodemographic details about the individuals, such as their gender, age, and occupation (Tokyo Metropolitan Area Transportation Planning Council 2013). The data were collected on Thursday, 1st October 2008, and hence represent a regular working day during the week, outside of all relevant holiday or festival periods. The questionnaire data had been further processed using various multimodal routing algorithms by a research group at the Center for Spatial Information Science (CSIS) at the University of Tokyo, who synthesized it into point positions in one-minute intervals (Usui et al. 2009; Sekimoto et al. 2011).

While this massive person trip data set allows for the analysis of person flows and singular trips, we filtered it for those time spans of stationarity between trips, where the individuals were not moving in space. Since these stationarity events themselves were not assigned a purpose in the data set, we classified them according to the purpose of the immediately preceding trip. This allowed us to extract the number of people in each location at each point in time pursuing in each of our six categories. It is important to note here that in the upcoming stage of the estimation process two activities represent the six categories we used so far: the activity "home" corresponds to the category "home," while the activity "work" contains the employees of all five remaining categories ("business \& office," "education," "retail \& service," "leisure \& hotel," and "public institution"). People engaging in these activities outside of their occupation, for example as customers of a shop or guests of a restaurant, cannot be captured by this method and are not contained in the resulting population estimation figures.

For privacy reasons the team at CSIS had to anonymize the exact spatial locations of the addresses provided in the original questionnaires. Therefore we were not able to assign the locations of the stationarity events (or the last location of the preceding trip) to the exact building locations, which would have made the subsequent population estimation process significantly simpler. Instead, the stationarity locations of all individuals within one areal unit, the so-called "person flow zones," had been generalized to one random location within that areal unit. It is worth mentioning that these person flow zones are not identical to the census tracts used by the population and employment census data sets, and are generally larger in area. Again, the fact that at this point in the estimation workflow we have already established categorical population figures for each building makes the use of such different spatial units possible.

In order to make the information about the stationarity events useable in the context of our estimation methodology we aggregated the number of distinct stationarity events within each person flow zone to hourly time steps (ranging from 0 to 23). We did this by counting each event from the time step it started in until the time step it ended in. These aggregated data can be understood as an hourly census of people within the sample population pursuing each activity within each person flow zone.

By defining the maximum population per category and person flow zone over 24 hours as a $100 \%$ index we went on to calculate which proportion of this maximum population was present in each person flow zone at each given point in time. This relative population ratio can be plotted as a graph showing the temporal fluctuation of population within each person flow zone, census tract or even building, per activity over time. Figure 5 shows an example of such data for a census tract on the artificial island of Tsukishima, which is characterized by a comparatively large residential population. It clearly shows how most people leave their homes in the morning and return in the evening. It also reveals the main working hours from roughly 8 a.m. and 9 a.m. to between 5 p.m. and 7 p.m. and thereby proves both assumptions we made in the previous section. 


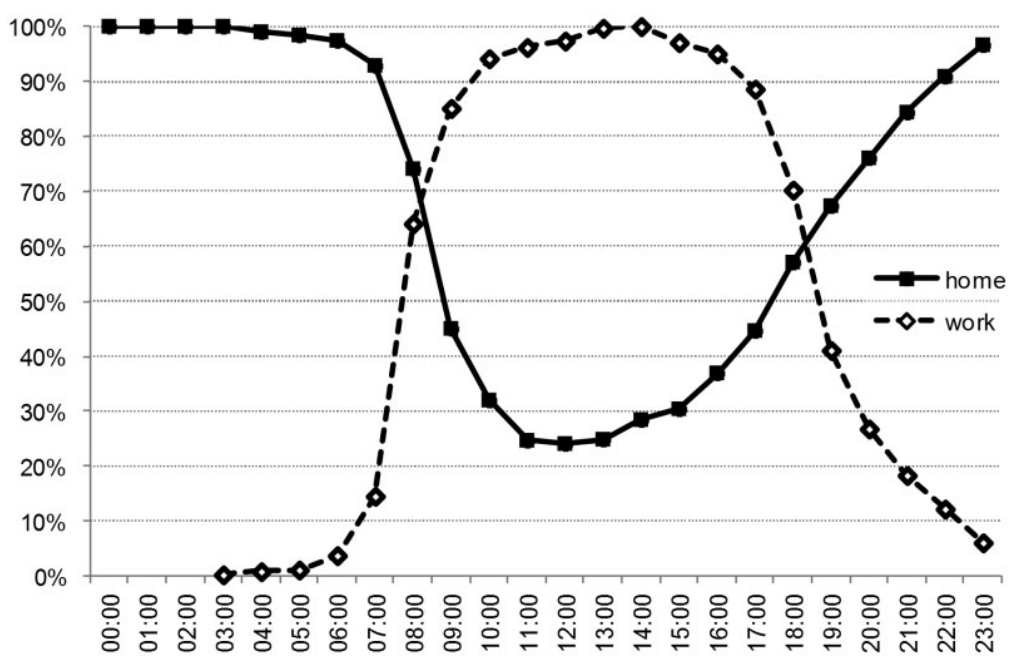

Figure 5 Gradients of the relative populations for the activities "home" ( $\mathrm{n}=506)$ and "work" ( $\mathrm{n}=$ 343) over 24 hours within one exemplar census tract of the study area ( $3^{\text {rd }}$ block of Tsukishima)

In the second to last step we combined the output data of these calculations with the output result of the penultimate step in the previous section. We assumed that the temporal variations of the proportional populations for each category, which we just calculated, are valid for everything within the respective person flow zone. In other words, if a person flow zone has its maximum working population at $10 \mathrm{a} . \mathrm{m}$. and only $50 \%$ of that population at 5 p.m. the same is assumed to be the case for all buildings within this person flow zone. In terms of calculation we applied the percentage of the total population per activity at each time step within each person flow zone to the estimated categorical population figures of each building. Figure 6 shows the results for the activities "home" and "work" at two different times of the day. It clearly reveals that a large number of people enter the study area during the day to pursue work there.

In a final step we then added up the temporally corrected estimated population figures of the two activities "home" and "work" for each building and each time step. This allows us to analyze the estimated total number of people per building over time. Figure 7 shows a time series of maps for the total building populations within the study area over 24 hours.

\section{Results}

\subsection{Validation}

Since no data about the actual building populations are available, the model output is difficult to validate. The only viable option appeared to be a manual count of the numbers of people entering and leaving a number of selected buildings, which allows for the calculation of the building populations.

The data were collected for seven hours each, between 7 a.m. and 2 p.m. on the 2 nd of July (buildings A and B) and on the 3rd of July (building C) 2013. All three buildings are located in the area around Tokyo Station. In their selection we paid close attention to the number and locations of entrances, i.e. the number of doors as well as the existence of 


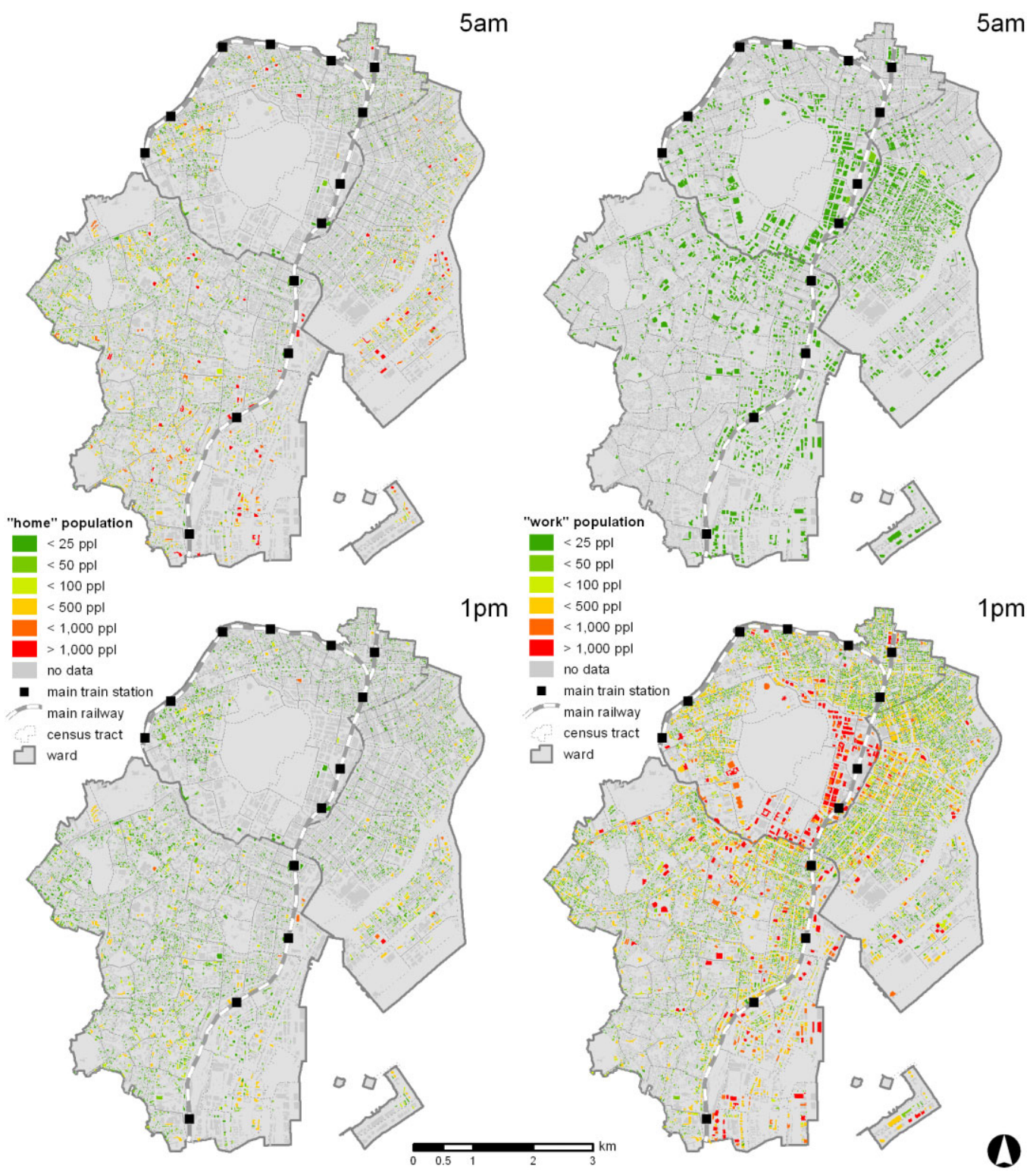

Figure 6 Map of the total estimated building population of each building pursuing the activities "home" (left) and "work" (right) at 5 a.m. (top) and 1 p.m. (bottom)

underground passageways and parking garages. These features, more precisely their nonexistence, severely limited the number of applicable buildings. Also the small number of buildings and the brief observation times are due to the limited time and resources available. Even with these limitations we deem the validation imperative for a meaningful discussion of the usefulness of our proposed estimation approach. To our best knowledge this is the first published validation of a spatio-temporal micro-scale population estimation model. 

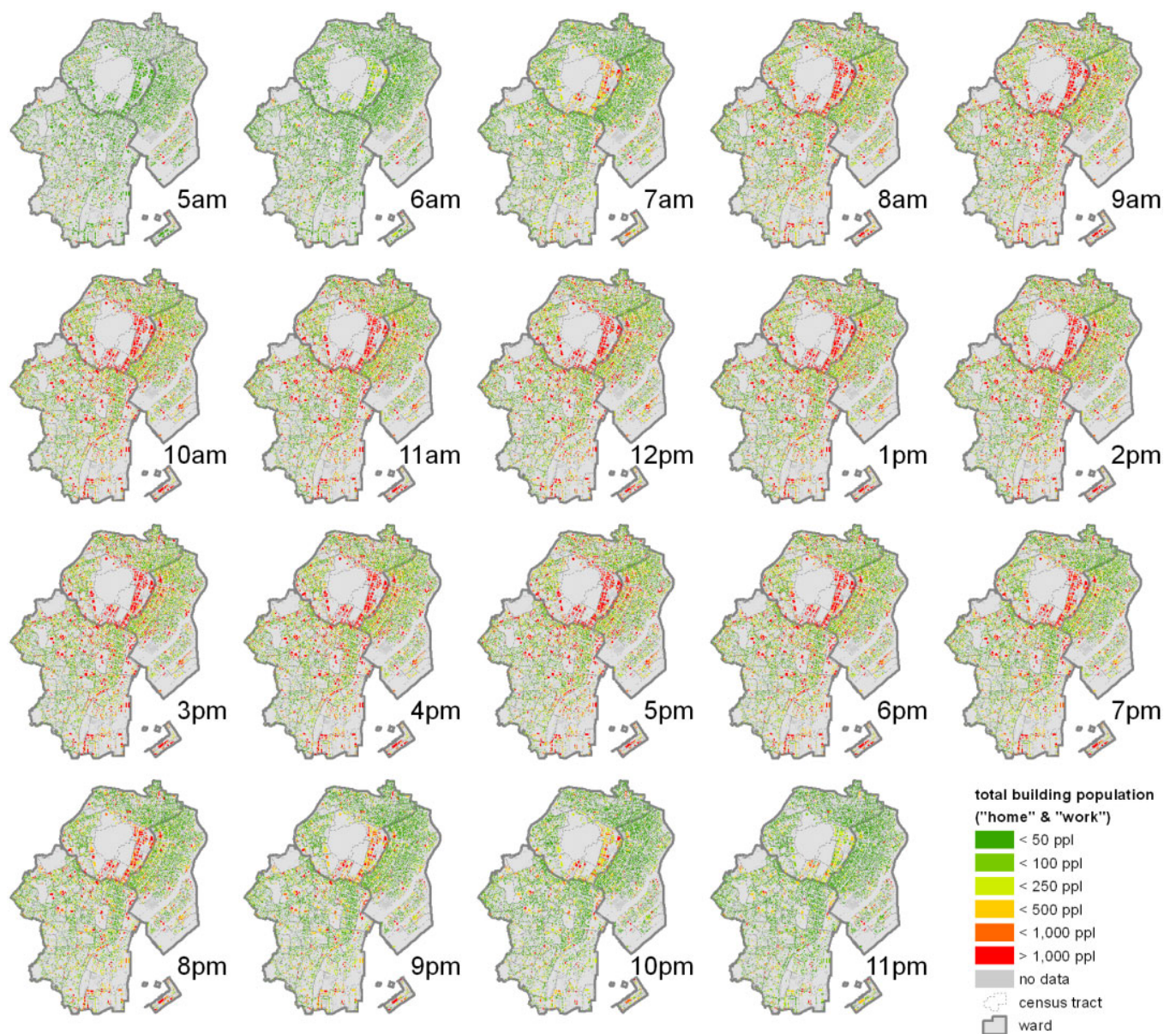

Figure 7 Time series maps of the total estimated building population of each building over time (5 a.m.-11 p.m.)

Building A is purely an office building with one tenant over ten floors and only one door. Building B is an eight-story mixed-use building with five offices, one retail store, a restaurant, and a total of three doors. Building $\mathrm{C}$ is a high-rise office building with 26 floors containing 39 offices, five shops, six restaurants, and a total of six doors. It is worth noting that we did not include buildings that contain residential uses, since the number of people inside the building at the start of our count would not be obvious. The same is true for employees who worked overnight or arrived before $7 \mathrm{a} . \mathrm{m}$. but, due to the domestic nature of the companies in these three buildings, we deem this effect negligible. While the model overall produces results close to the actual measured building populations, there are some obvious variances, which we explain in the following section.

The employees of all buildings arrive later for work than our model predicted, mostly between 7 a.m. and 8:59 a.m., therefore the model greatly overestimates the number of people present in the buildings for those times. As Figure 8 shows, the deviations are $340 \%, 3,600 \%$ (not shown in the graph), and 503\%, respectively. We attribute this to the fact that the movement data we use in our methodology had originally been collected using paper 


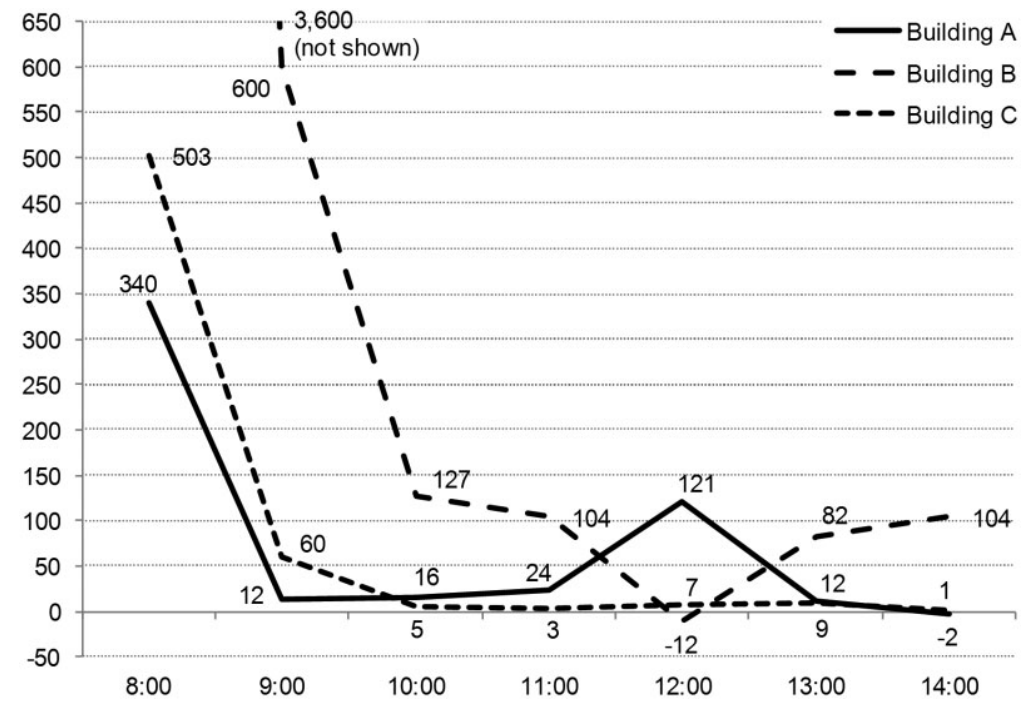

Figure 8 Over- and under-estimation percentages of the population estimation model compared to the actual observed building populations of the sample buildings

questionnaires. The given start and end times of trips therefore do not necessarily represent the precise times in reality, but rather the more generalized perceived or memorized times. This becomes obvious in the structure of the time stamps that represent the start of work for the sample individuals. A detailed analysis of the underlying data shows that $88 \%$ of those stationary working activities supposedly started at round-numbered minutes such as ":00," ": 10 ," ": 15 " etc. $27 \%$ were apparently started exactly at the full or half-hour marks. This becomes especially problematic when we group these numbers by hour. If, for example, an employee started working at 8:57 a.m. but entered 9:00 a.m. in the questionnaire, he would fall into the $8 \mathrm{a} . \mathrm{m}$. group in our door count, but in the 9 a.m. group in the movement data.

Buildings $\mathrm{A}$ and $\mathrm{C}$ show comparatively high numbers of people leaving these buildings during the whole observation period and from as early as 9 a.m. These numbers can be attributed to short-term visitors, who entered the buildings for the purpose of business meetings or deliveries. In the case of building $\mathrm{C}$, another major factor is the existence of a convenience store and a coffee shop on the ground floor, which attracted large numbers of customers starting from 8 a.m.

Figure 8 shows the over- and under-estimation of the model in percent compared to the actually observed numbers for all three buildings. In comparison to the other two, building B generally shows a higher deviation. This can be attributed to the comparatively small number of people in the building, which amplifies the model's uncertainties. The other two sample buildings are within $60 \%$ deviation from 9 a.m. and below $25 \%$ after 10 a.m. (building C even below $5 \%$ ), with the exception of the lunch hour between 12 p.m. and 1 p.m. Here building A is greatly overestimated $(+121 \%)$, which can be attributed to the coarse temporal resolution of hourly steps in the modeling process. People who leave and re-enter the building within a short time span (such as a 30-minute lunch break) cannot be covered by the model, which looks only at the two activities of "home" and "work." If, for example, employees leave their workplace at 12:30 p.m. they will be counted as pursuing the activity "work" for the hour from 12:00 p.m. to 12:59 p.m. If they return by 1:15 p.m. they will again be counted 
as pursuing the activity "work" for the hour from 1:00 p.m. to 1:59 p.m. The fact that they have actually left the building in the meantime is not reflected in the model, but only in the door count. The same effect can also be seen in the numbers of building B, albeit in the opposite direction: here we counted a great number of people entering the building in the time from 12 p.m. to 1:59 p.m. to have lunch in the restaurant there. Since these customers do not show up in the model, it underestimates the actual number by $12 \%$. Building $\mathrm{C}$ does not show either of these two effects to a greater degree, since the number of employees leaving the building during lunchtime and the number of customers entering the building to have lunch in one of the building's restaurants almost even each other out.

Ahola et al. (2007, p. 950-51) mention that "more detailed information about the temporal behaviour of different population groups could also improve the quality of the model". We believe that the inclusion of spatio-temporal movement data of a large population sample achieves this improvement, as it provides data about the exact locations of each individual at any time and ameliorates the a priori assumptions that Ahola et al. (2007) had to rely on in their modeling approach. We therefore believe our model to be able to indicate the actual changes in building populations over time more precisely than previous approaches have been able to. Nevertheless, the modeling accuracy could be further improved by using a finer timescale, e.g. 30-minute, 15-minute or even 1-minute intervals, and by including short-term populations from the remaining four activities, such as customers, guests, and visitors in the modeling process.

\subsection{Discussion}

The enhanced spatio-temporal building population estimation approach we introduced in the preceding section can be used to produce a variety of output data and products. Firstly, the geolocated address point data allow for an overview of the spatial distribution of different activity categories and the locations that facilitate these activities (Figure 2). While this is not an outcome of the estimation model as such, we would like to emphasize how even the simple first step of defining broad activity categories and the visualization of just one data set can provide a meaningful insight into the urban structure defining the spatio-temporal effects the model elaborates on in its further steps.

Secondly, the first part of our proposed model extends the preliminary estimation approach by a number of usage categories over the one-dimensional focus on residential building populations. This alone can help to get a more realistic representation of the population of each building in a study area. As we mentioned above, this is especially important when analyzing areas whose primary land use is not residential. A prime example is highly urbanized city centers, which are characterized by a multitude of different land uses gathering in close spatial proximity to each other, and often even mixed within single buildings. Our enhanced approach covers these peculiarities and maps the underlying real-world processes to the buildings under analysis. A closer look at the spatial distribution of buildings with a high number of usage categories reveals that they are mainly clustered in the business districts, which are also the locations of most of the high-rise buildings in the study area (e.g. in Marunouchi west of Tokyo Station, around Shinbashi, Tamachi, and Shinagawa Stations, and in Akasaka in the northern Minato ward). These agglomerations of multiple usage categories can be understood as a result of the need for multiple activities in the close surroundings of the workplace. These usage mixes allow employees to perform all their daily routine activities within one building, which is convenient in terms of both travel time and cost. 
Thirdly, the introduction of the temporal dimension to the estimation of building populations allows for a micro-scale analysis of the actual population figures according to the underlying human activities and the data sets used in the process. We believe that this is the most interesting characteristic of our proposed estimation methodology, since for the first time it allows a reliable estimation of building populations even for large study areas with justifiable requirements in terms of both necessary input data and computational expense. We formulated the calculations shown in the two sections above as a series of SQL statements that process a multitude of tables within a PostgreSQL database with the PostGIS extension installed to make use of geographical functionalities. On a contemporary standard laptop computer the complete spatio-temporal building population estimation for ca. 51,000 buildings in the study area took roughly 1.5 hours.

The output result of the spatio-temporal model can be used in a multitude of ways. Examples for visualizations are graphical representations of the population figures over time for single buildings or census tracts (Figure 5), and maps of the population distribution at a certain point in time (Figure 6) or as time series maps (Figure 7). In addition, the output data can be used for further quantitative analyses, such as population density calculations for certain points in time. They can also be used as input for other quantitative models, such as traffic volume estimations in an urban planning context, or customer volume estimation models in an economic geographical context. In a risk and hazard context these micro-scale building populations could be used to identify realistic starting scenarios for multi-agent-based tsunami evacuation simulation models like those introduced by Mas et al. (2013).

But it is also interesting to have a detailed look at the output data themselves, since several interesting trends and facts can be identified therein. Due to the aforementioned spatial generalization of the person trip data we are not able to make detailed explanations about the temporal characteristics of single buildings, but a look at the types of buildings and their respective person flow zones, to which the person trip data had been generalized, allows for some interpretations.

Figure 9 shows the temporal gradient of the sample populations from the person flow data set for both the residential and working activities. Most of the gradients follow the same patterns, with only small deviations. The major variances in Figure 9a are the result of the small residential sample populations in person flow zones 4, 5, 7, and 18 . We marked them with dashed lines to emphasize this circumstance.

Firstly, employees in person flow zone 10 (annotated in Figure 9b) appear to arrive at work earlier than those in the remaining zones. An explanation for this is the Tokyo Metropolitan Central Wholesale Market, more commonly known as the "Tsukiji Fish Market." Secondly, there are obviously no big differences between the person flow zones during the working hours of the day. All zones cross the $90 \%$ mark in terms of the relative working population between 9 a.m. and 10 a.m. and stay on this level until 5 p.m. (Figure 9b). This can be explained by the relatively equal spatial distribution of offices across the study area, as was shown in Figures 2 and $3 \mathrm{~b}$. While the common working hours in Japanese offices are from 9 a.m. to 5:40 p.m., the scheme of flexible working hours is getting more and more popular in Japan too, which may explain some of the early starters in person flow zones 3, 4, 22, and 24 . These contain the business districts of Marunouchi/Otemachi west and northwest of Tokyo Station, as well as those around Shinagawa Station, Shiodome/Shinbashi Stations, and Tamachi Station. They are characterized by a large number of high-rise office towers, which we define as buildings over 15 floors: $92,49,88$, and 47 , respectively, which accounts for $46 \%$ of all high-rise buildings in the study area. These contain many offices of larger companies, which in turn are more prone to offer their employees the aforementioned flexible working times. 

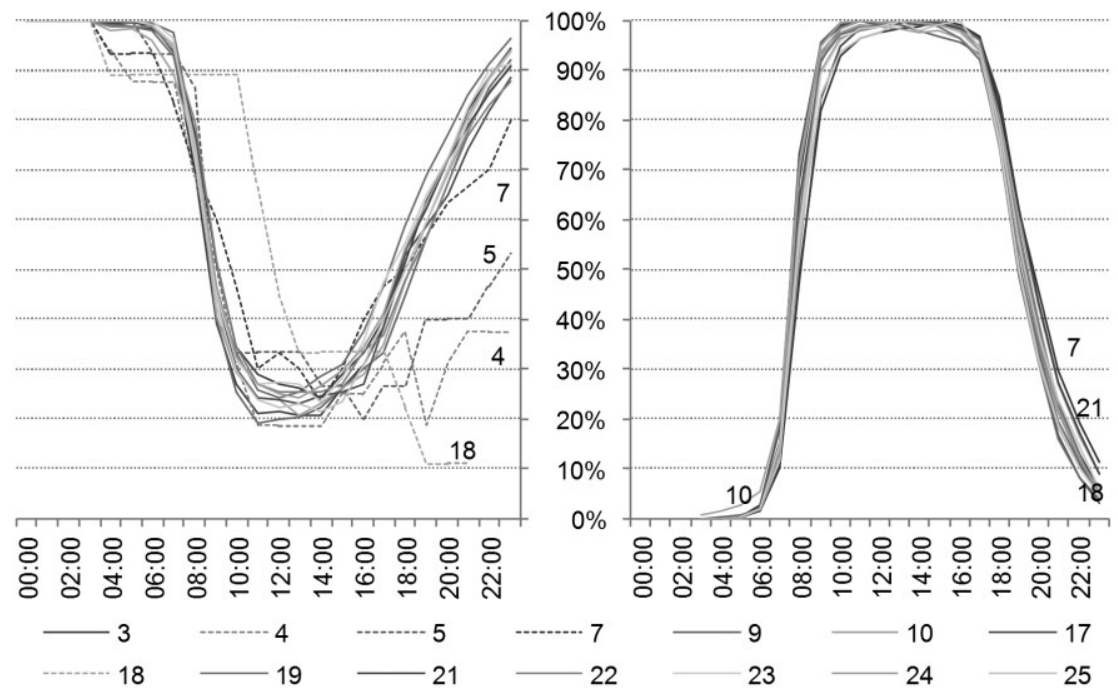

Figure 9 Temporal gradients of the sample populations for each person flow zone in the activities "home" (left) and "work" (right)

Lastly, late at night three person flow zones (annotated in Figure 9b) stand out due to their comparatively higher levels of employees until after 7 p.m. and even later after 10 p.m. The area of the $5^{\text {th }}, 6^{\text {th }}$, and $7^{\text {th }}$ blocks of Ginza between Sotobo Street and Chuo Street in person flow zone 7 is characterized by a large number of restaurants, bars, and other leisure establishments $(3,947$ in total), which require employees to work until late. Conversely, the area west of Hibiya Park (the southernmost part of Chiyoda ward) in person flow zone 18 is the home of multiple ministerial buildings and other governmental institutions, which require the attendance of a number of employees at any time during night and day. Along the same lines, the high number of late-night workers in person flow zone 21 can be explained: the office complex “Tokyo Midtown" near Roppongi houses a number of international corporations, whose offices are staffed at night due to the necessary interactions with co-workers and customers in different time zones. In addition, the area of the $3^{\text {rd }}$ block of Akasaka has a large number of bars and restaurants (1,130 in total), which require service personnel late at night similar to the aforementioned district in Ginza.

Thereby we identified four real-world concepts or processes in the output of our spatiotemporal modeling: (1) the early start of operations at the big wholesale market; (2) the adoption of flexible working hours in larger corporations; (3) the necessity for late-night office attendance due to globalized business activities; and (4) the Japanese custom of visiting restaurants and bars near the workplace after work on weekdays as well.

\section{Conclusions}

In this article, a new approach was introduced for the spatio-temporal estimation of building populations in highly urbanized areas. It extends a number of previously published population estimation approaches (Martin 1996; Elvidge et al. 1997; Tobler et al. 1997; Dobson et al. 2000; Sutton et al. 2001; McPherson and Brown 2004; Ahola et al. 2007; Bhaduri et al. 2007; 
Lwin and Murayama 2009; Martin et al. 2009) through the differentiation of two activities ("home" and "work") and by operationalizing the temporal dimension of human activities using a very detailed movement profile data set. The former allows for a more realistic representation of human activities in urban areas, while the latter accounts for the fact that populations change not only spatially but also over time, as a result of the aforementioned activities. For the first time, our approach allows for a temporal modeling that is more detailed than the diurnal separation of daytime and nighttime populations, and that also does not rely on a priori assumptions and expert judgement regarding the time profiles of human activities.

The preliminary approach by Lwin and Murayama (2009), on whose areametric population estimation methodology we built, is well suited for the analysis of suburban or rural areas, where the main usage can be considered residential, hence making the inclusion of other activities unnecessary. By contrast, highly urbanized areas are characterized by the co-occurrence of a variety of land uses, such as residential, commercial, educational, recreational, etc., often even mixed within buildings. The population estimation approach we introduced in this article accounts for this fact by incorporating both the differences in the usage of buildings and the temporal variations in human activities within highly urbanized areas.

We have furthermore developed a computational model that implements these variances over space and time. The model makes use of a variety of micro-scale data sets to operationalize the aforementioned activities and their spatio-temporal representations. The outcome of the model provides an estimated population figure for each building and at each time step. In doing so, the model reveals spatio-temporal behavior patterns inherent in the data that could otherwise not have been visualized. The output can be used in a variety of applications regarding the implications of human behavior in urban areas, such as urban planning, transportation geography, and risk analysis, to name only a few.

For the first time we also conducted a validation of our model data by comparing it to a real-world door count for a number of sample buildings within our study area. This validation has shown some shortcomings inherent in the model. So far the spatio-temporal model has only taken into consideration two activities: "home" and "work." None of the other daily routine activities pursued by the people in highly urbanized areas, such as education, shopping, recreation, daily errands, etc., are reflected. This effect became obvious in the low accuracy during the office lunchtime hours between 12 p.m. and 1:59 p.m., where the building populations were greatly under- or overestimated according to the existence or non-existence of restaurants in the respective buildings. So far the model has focused only on the more longterm activities (with the notable exception of "education"), where people would stay within the same building over an extended amount of time. These populations can be called "stationary populations." Short-term activities such as the aforementioned are completely neglected; the respective "transient populations" are not reproduced in the model. Therefore two main amendments to the model have to be the inclusion of: (1) "education" populations as the missing stationary population category; and (2) all remaining transient population categories, i.e. "shopping," "entertaining," and "errand".

In addition, model inaccuracies in the morning hours can be attributed to the collection method of the underlying movement data. Since these were obtained using paper questionnaires, a strong tendency to round-number minutes in the time stamps was introduced. In connection with the coarse temporal resolution of our population model, this led to severe estimation errors. Therefore, two more modifications to improve the model accuracy have to be: (1) the use of more temporally precise movement data, possibly collected in an automated process using GPS-enabled devices; and (2) the use of a temporal scale finer than the hourly 
intervals we used in this case study. We would also like to mention that the nature of the data we used in this research did not allow for a comparison of different temporal cycles, such as weekdays as opposed to the weekend, holiday periods vs. normal school and working terms, or seasonal changes, which would undoubtedly all provide further interesting insights into the spatio-temporal changes in human activity patterns.

On a different note, some shortcomings of the model in regard to the modeling precision can be attributed to the underlying data and their deficiencies. While all data sets used in the case study area are amongst the best available in their respective realms, they impaired the model calculation due to missing and incomplete data. In particular, missing address points, buildings with missing attribute information, and the aforementioned flaws of the person trip data have had significant implications on the overall model output. We are confident that it could be improved significantly if these deficiencies were amended. Also, several generalizations in our estimation model can possibly have an effect on the calculation results. These generalizations are inherent in the assumptions of: (1) an equal split of a building's floor space among the contained usage categories; and (2) equal floor space use within each usage category, neglecting differences among various residential types, as well as office and store layouts. Both can lead to skewed distributions and therefore biased outcomes but are impossible to quantify or eliminate given the available data.

It is generally to be desired that this approach be applied to different spatial contexts, i.e. other highly urbanized areas in Japan and other countries. This could enable some interesting comparative analyses between various types of urban agglomeration, their commonalities and differences.

\section{References}

Ahola T, Virrantaus K, Krisp J M, and Hunter G J 2007 A spatio-temporal population model to support risk assessment and damage analysis for decision-making. International Journal of Geographical Information Science 21: 935-53

Axhausen K W, Zimmermann A, Schönfelder S, Rindsfüser G, and Haupt T 2002 Observing the rhythms of daily life: A six-week travel diary. Transportation 29: 95-124

Bhaduri B, Bright E, Coleman P, and Urban M L 2007 LandScan USA: A high-resolution geospatial and temporal modeling approach for population distribution and dynamics. GeoJournal 69: 103-17

Bowman J and Ben-Akiva M 2001 Activity-based disaggregate travel demand model system with activity schedules. Transportation Research Part A: Policy and Practice 35: 1-28

Dobson J E, Bright E E, Coleman P R, Durfee R C, and Worley B A 2000 LandScan: A global population database for estimating populations at risk. Photogrammetric Engineering and Remote Sensing 66: 849-57

Elvidge C D, Baugh K E, Kihn E A, Kroehl H W, and Davis E R 1997 Mapping city lights with nighttime data from the DMSP Operational Linescan System. Photogrammetric Engineering and Remote Sensing 63: $727-34$

Horanont T 2012 A Study on Urban Mobility and Dynamic Population Estimation by Using Aggregate Mobile Phone Sources. WWW document, http://www.csis.u-tokyo.ac.jp/dp/115.pdf

Jiang S, Ferreira J, and González M C 2012 Clustering daily patterns of human activities in the city. Data Mining and Knowledge Discovery 25: 478-510

Lwin K and Murayama Y 2009 A GIS approach to estimation of building population for micro-spatial analysis. Transactions in GIS 13: 401-14

Malczewski J 1999 GIS and Multicriteria Decision Analysis. New York, John Wiley and Sons

Martin D 1996 An assessment of surface and zonal models of population. International Journal of Geographical Information Systems 10: 973-89

Martin D, Cockings S, and Leung S 2009 Population 24/7: Building time-specific population grid models. In Proceedings of the European Forum for Geostatistics Conference, The Hague, Netherlands

Mas E, Supparsi A, Srivihok P, Koshimura S, and Imamura F 2013 Feasibility of evacuation at the Pakarang Cape in Thailand based on tsunami inundation model and human evacuation simulation. In Proceedings of the Tenth International Conference on Urban Earthquake Engineering, Tokyo, Japan 
McPherson T N and Brown M J 2004 Estimating Daytime and Nighttime Population Distributions in U.S. Cities for Emergency Response Activities. Seattle, WA, American Meteorological Society

Schmitt R C 1956 Estimating daytime populations. Journal of the American Institute of Planners 22: 83-5

Sekimoto Y, Shibasaki R, Kanasugi H, Usui T, and Shimazaki Y 2011 PFlow: Reconstructing people flow recycling large-scale social survey data. IEEE Pervasive Computing 10: 27-35

Sutton P C, Elvidge C, and Obremski T 2003 Building and evaluating models to estimate ambient population density. Photogrammetric Engineering and Remote Sensing 69: 545-53

Sutton P, Roberts D, Elvidge C, and Baugh K 2001 Census from heaven: An estimate of the global human population using night-time satellite imagery. International Journal of Remote Sensing 22: 3061-76

Tobler W R 1979 Smooth pycnophylactic interpolation for geographical regions. Journal of the American Statistical Association 74: 519-30

Tobler W, Deichmann U, Gottsegen J, and Maloy K 1997 World population in a grid of spherical quadrilaterals. International Journal of Population Geography 3: 203-25

Tokyo Metropolitan Area Transportation Planning Council 2013 パーソントリップ調査とは (About the Persontrip Study). WWW document, http://www.tokyo-pt.jp/person/index.html

Usui T, Kanasugi T, Sekimoto Y, Minami Y, Shibasaki R, and Nanako A 2009 Realization and implementation of Tokyo Metropolitan Area person-trip data spatio-temporal interpolation by a People Flow Analysis Platform. Papers and Proceedings of the Geographic Information Systems Association 18: 541-45 (in Japanese)

Wu S, Qiu X, and Wang L 2005 Population estimation methods in GIS and remote sensing: A review. GIScience and Remote Sensing 42: 80-96

\section{Supporting information}

Additional Supporting Information may be found in the online version of this article at the publisher's website:

Figure A1 Map of the person flow zones of the study area in relation to the census tracts.

Figure A2 Outcome of the validation of the model estimation using door counts for three sample buildings: (a) A; (b) B; and (c) C. 